\title{
Experimental study of the separation behavior of nanoparticles in micro- and nanochannels
}

\author{
Mariateresa Napoli • Paul Atzberger • \\ Sumita Pennathur
}

Received: 11 March 2010/Accepted: 19 May 2010/Published online: 10 June 2010

(C) The Author(s) 2010. This article is published with open access at Springerlink.com

\begin{abstract}
In this article, we investigate the effects of $\mathrm{pH}$, ionic strength, and channel height on the mobility and diffusivity of charged spherical particles within planar microfluidic channels. Specifically, we report results of a broad experimental study on the transport and separation behavior of 50 and $100 \mathrm{~nm}$ spherical carboxylated polystyrene nanoparticles, confined in $20 \mu \mathrm{m}, 1 \mu \mathrm{m}$, and $250 \mathrm{~nm}$ deep fluidic channels. We find that $\mathrm{pH}$, ionic strength, and channel height have coupled impacts on mobility changes. In particular, we show that, depending on $\mathrm{pH}$, the dependence of particle mobility on channel size can have opposing behavior. In addition, we also show that at the nanoscale, at lower ionic strengths, there is a substantial increase in mobility, due to enhanced electric fields within the nanochannels. These effects are important to understand in order to avoid potential downfalls in terms of separation efficiency as well as design for better tuning of separation performance in micro- and nanochannels. Finally, we propose a method to estimate the effective zeta potential of spherical particles from measured electrophoretic mobility data. This could prove useful in characterizing a heterogeneous collection of particles having a distribution over a range of values of the zeta potential.
\end{abstract}

M. Napoli $(\bowtie) \cdot$ P. Atzberger · S. Pennathur

Department of Mechanical Engineering, University

of California, Santa Barbara, CA, USA

e-mail: napoli@engineering.ucsb.edu

URL: http://www.engineering.ucsb.edu/ nanolab/

P. Atzberger

e-mail: atzberg@math.ucsb.edu

S. Pennathur

e-mail: sumita@engineering.ucsb.edu

P. Atzberger

Department of Mathematics, University of California,

Santa Barbara, CA, USA
Keywords Spherical nanoparticles .

Micro- and nanoscale electrophoresis · Wall effects

\section{Introduction}

In the past decade, advances in micro- and nanofabrication techniques have contributed to the rapid development of the field of micro- and nanofluidics. This field holds great promise for the realization of portable bioanalytical tools for medical and more general sensing applications. Specially in view of novel medical applications for disease diagnosis, there has been a remarkable increase of investigations focused on biomolecule separations in planar microfabricated channels, fueled by the potential of integrating these channels into portable diagnostic devices. While there has been significant recent progress on the experimental characterization of such systems, precise mechanisms involved with micro- and nanofluidic separations of biomolecules remains only partially understood. This is due in part to the nature of biomolecules, which can have complex interactions with each other as well as with their environment. The overwhelming complexity of individual biomolecules in terms of their charge and conformational states has led many researchers toward finding a simpler model system with which to test general hypothesis and trends. Spherical polystyrene nanoparticles can serve as such a simplified model system, offering to a first approximation some insights into the possible dynamics in channels of more complex biomolecules (e.g., short strands of DNA, globular proteins) (Benke et al. 2008). These studies could help uncover relevant physical phenomena that govern experimentally observed behavior of biomolecules when confined to microchannels and nanochannels. 
Analytical studies of electrophoretic particle transport date back at least to the 1970s (Morrison 1970; Dukhin and Derjaguin 1974; Hunter 1981). These classical studies consider the case of particles suspended in an unbounded electrolyte solution and show that, in the thin electric double layer (EDL) limit, the Smoluchowski equation (Smoluchowski 1921; Huckel 1924) applies to particles of arbitrary shape. Henry's correction function further allows these results to be extended to the case of particles with a nonnegligible EDL, by introducing a scaling factor that depends on particle size and Debye length (Henry 1931; Ottewill and Shaw 1972). For microfluidic applications, the treatment of the system as an unbounded domain may not always be a good approximation. It has been shown that the presence of a channel wall can affect the particle electrophoretic motion by coupled hydrodynamic and electrostatic effects. For example, in the thin EDL limit, theory predicts that a particle electrophoretic velocity is reduced by nonconducting channel walls (Keh and Anderson 1985; Keh and Chiou 1996; Yariv and Brenner 2002; Yariv and Brenner 2003b). An experimental investigation that supports this theory can be found in Xuan et al. (2006), where the authors study wall effects in microchannels on the electrophoretic motion of polystyrene particles of comparable size. In this work, the authors use a convergingstraight-diverging PDMS channel and find that larger particles are retarded at the sidewalls of the channel due to viscous forces, which is in good agreement with theoretical results. When the particle-wall separation decreases further, theory predicts an increase in particle electrophoretic velocity (Keh and Chen 1988; Keh and Chiou 1996; Yariv and Brenner 2003a; Majumder et al. 2007). Furthermore, Qian et al. (2006) theoretically study the electrophoretic motion of a spherical particle in a nanotube with a converging-diverging section, finding that particle electrophoretic mobility becomes spatially dependent, a mechanism that the authors propose to enhance particle separation (Xuan et al. 2005). In the limit of thick EDL, it was found theoretically that wall effects become more significant (Ennis and Anderson 1997; Shugai and Carnie 1999) and in general that particle shape matters, but that the effects are similar to the case of spherical particles (Hsu and Kao 2002; Ye et al. 2002; Liu et al. 2004).

Besides confinement, the $\mathrm{pH}$ also has an important effect on particle transport. A number of papers have dealt with the influence of $\mathrm{pH}$ on the $\zeta$ potential of microfluidic channels (Schwer and Kenndler 1991; Kosmulski and Matijevic 1992; Scales et al. 1992; Dickens et al. 1994; Caslavska and Thormann 2001; Kirby and Hasselbrink 2004). In Kuo et al. (2001) and Schoch and Renaud (2005), the authors show how transport of ions through nanoslits or nanoporous membranes can be tuned by adjusting the $\mathrm{pH}$. For the case of particles, most of the studies have been concerned with the impact of $\mathrm{pH}$ on aggregation and adsorption (Guzman et al. 2006; Ghosh et al. 2008). Two early papers consider explicitly the effect of $\mathrm{pH}$ on the electrophoretic mobility of latex nanoparticles, but also in the context of investigating colloid stability and understanding the factors impacting it (Maron and Bowler 1948; Maron et al. 1948).

In this work, we perform a parametric experimental study on the transport and separation behavior of spherical, charged, polystyrene nanoparticles (50 and $100 \mathrm{~nm}$ diameter), within electrokinetic flows in micro- and nanochannels, under different experimental conditions of buffer $\mathrm{pH}$, ionic strength, and channel geometry. We show that particle electrophoretic mobility depends on all of these parameters, presenting a marked difference in behavior between micro- and nanochannels that can impact their efficacy as separation devices. We discuss the origin of the observed behavior in terms of known theoretical results and point out experimental discrepancies that have yet to be explained. We also discuss our thoughts on some possible hypotheses, which might explain these discrepancies.

\section{Experimental materials and methods}

\subsection{Chemicals and reagents}

Carboxylated polystyrene nanoparticles (Fluoresbrite ${ }^{\circledR}$ Polysciences, Inc.), 50 and $100 \mathrm{~nm}$ in diameter, were suspended at $50 \mu \mathrm{g} / \mathrm{l}$ concentration, with the addition of $0.1 \% \mathrm{v} / \mathrm{v}$ Tween 20 (Sigma-Aldrich, St. Louis, MO), to mitigate aggregation and adsorption problems. This concentration of particles corresponds to approximately $10^{8}$ particles per $\mu \mathrm{l}$, resulting in a very low volume fraction, e.g., a dilute suspension. Reagents included buffered solutions consisting of acetate $(\mathrm{pH} 5)$, phosphate $(\mathrm{pH} \mathrm{7})$, and borate $(\mathrm{pH} 9)$ at two concentration values, $1 \mathrm{mM}$ and $10 \mathrm{mM}$. All solutions were filtered, prior to use, with $200 \mathrm{~nm}$ pore syringe filters (Nalgene, Rochester, NY).

\subsection{Microfluidic device and setup}

Three types of standard, fused silica microfluidic devices were fabricated for this study, simple cross, isotropically etched fluidic chips: $20 \mu \mathrm{m}$ deep (Micralyne Inc., Alberta, Canada), $1 \mu \mathrm{m}$ deep (Micronit, Enschede, the Netherlands), and $250 \mathrm{~nm}$ deep (Dolomite Ltd, UK). All channels had a separation column length of at least $30 \mathrm{~mm}$. Figure 1a shows a schematic of the channel geometry and our naming convention for each well: $\mathrm{N}$ (northern), $\mathrm{S}$ (southern), W (western), and E (eastern). Electrical potentials were applied at these wells using platinum electrodes (Omega Eng. Inc., Stamford, CT), connected to a high 
voltage power supply (LabSmith HVS448). The pre-programmed voltage scheme for sample loading and injection was designed following the recommendations of Bharadwaj et al. (2002). Briefly, during the loading phase, the sample solution is placed in the $\mathrm{N}$ well and electrodes in the $\mathrm{N}, \mathrm{W}$, and $\mathrm{E}$ wells are set to positive voltages, while the $\mathrm{S}$ well is grounded, resulting in electrokinetic flow from all wells toward S. For the injection step, the applied voltages are then switched (W, N, S at high, E at ground), and the sample is injected along the $\mathrm{E}$ channel. The resulting electric fields during injections ranged from about 1.5 to $15 \mathrm{kV} / \mathrm{m}$. In particular, for each combination of experimental parameters $(\mathrm{pH}$, ionic strength, channel type, detection point), we applied three electric field values during injection, corresponding to multiples of a nominal value $(1 \times, 5 \times, 10 \times$ for $20 \mu \mathrm{m}$ deep channels; $1 \times, 2 \times, 4 \times$ for $1 \mu \mathrm{m}$ deep, and $250 \mathrm{~nm}$ deep channels). In between injections, the channel was flushed by applying a voltage scheme corresponding to the loading step. For the $20 \mu \mathrm{m}$ deep channels, we inserted removable reservoirs at the wells to mitigate pressure gradients. More specifically, particles were loaded into the channel and the level of solution in the reservoirs was adjusted so that, when the electric fields were switched off from the loading configuration, only diffusion (and no net particle motion) was observed at the channel intersection.

\subsection{Injection experiments}

Before introducing any new buffer solution (type or concentration), the channels were rinsed 20 min with filtered deionized water, followed by a $5 \mathrm{~min}$ rinse with $10 \mathrm{mM}$ $\mathrm{NaOH}$ solution, followed by a final 20 min rinse with filtered deionized water. The voltage scheme used corresponds to the loading configuration described above.
Transport data were obtained recording fluorescence intensity images of the particles within the channels, using an epiffuorescent microscope (Olympus IX71), fitted to an EMCCD camera (Andor Inc, iXon) and either a $20 \times$ or a $60 \times$ oil immersion objective (see Fig. 1b). For more details on this now common acquisition method, see Mosier (2001), Bharadwaj et al. (2002), and Pennathur and Santiago (2005b). These fluorescence images were then analyzed using Matlab (The MathWorks). Figure 1c shows a schematic of our data post-processing algorithm: we first crop the images to isolate the portion corresponding to the channel (see dashed horizontal lines in Fig. 1c); each four pixels in the longitudinal $(x)$ channel direction are assigned the intensity value corresponding to the average computed across the channel width $(y)$ in the corresponding four columns of pixels (schematically indicated as "average area" in the figure). With this procedure, we transformed bidimensional image data into typical plots of intensity versus time (electropherograms) and were able to extract the parameters of interest, such as time-to-arrival, which is a direct measurement of area-averaged velocity, and diffusion coefficients, from the Gaussian fits of electropherograms. Each measurement was repeated at least three times at each observation point $(5,10$, and $20 \mathrm{~mm}$ for $20 \mu \mathrm{m}$ deep channels, $6.5 \mathrm{~mm}$ for $1 \mu \mathrm{m}$ deep channels, and $5 \mathrm{~mm}$ for $250 \mathrm{~nm}$ deep channels) and for each combination of experimental parameters (e.g., pH, ionic strength, electric field, channel type) to check consistency and repeatability, for a total of more than 600 measurements.

\section{$2.4 \zeta$-potential measurements of the channel}

To estimate the $\zeta$-potential of the channel surfaces, $\zeta_{\mathrm{w}}$, we injected fluorescein into our fluidic channels and used Helmholtz-Smoluchowski relation to extract the value of
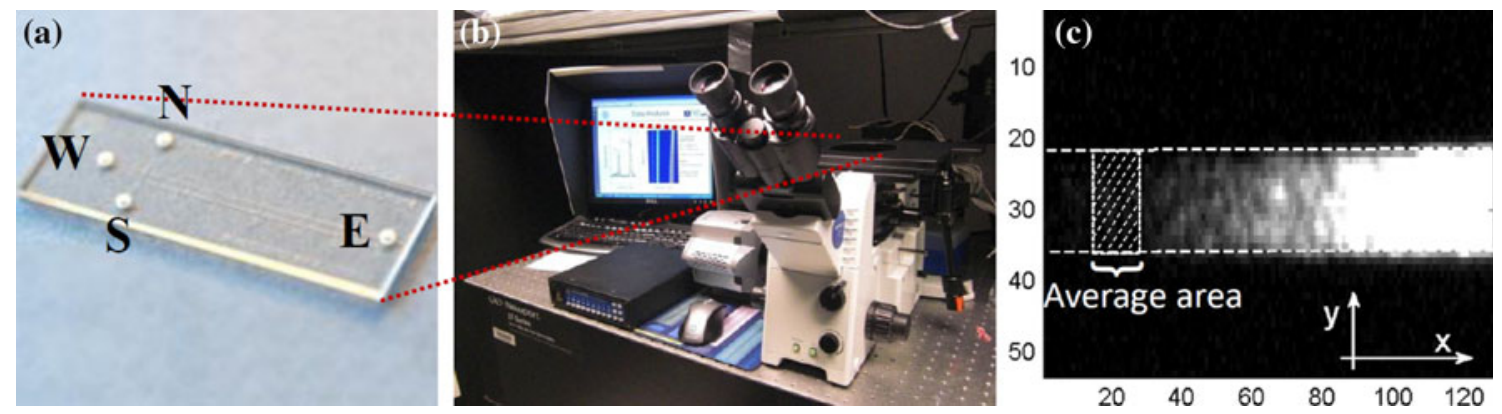

Fig. 1 Images of our electrokinetic experimental setup and schematic of data post-processing: a the nanofluidic channel is placed on top of the objective of an inverted microscope. The symbols N, S, W, and E denote the wells as, respectively, North, South, West and East; b picture of the actual setup, consisting of a high voltage supply (LabSmith, HVS448), an epi-fluorescent microscope (Olympus, IX71), with attached mercury bulb and back-illuminated EMCCD camera (Andor, iXon +); c example of a typical fluorescence image and schematic of data post-processing: tickmarks denote row and column number for the matrix of pixels corresponding to the image; dashed lines marking channel width denote cropped area and have been added to facilitate image interpretation. Each four pixels in the $x$ direction are assigned the intensity value corresponding to the average computed across the channel width, for the corresponding four columns (dashed region denoted as "average area", exaggerated in dimension for ease of reading) 
$\zeta_{\mathrm{w}}$, assuming thin EDL, from the measured time-to-arrival of fluorescein, $t_{F \mathrm{~L}}$, at each electrolyte concentration and $\mathrm{pH}$ used in this study:

$t_{\mathrm{FL}}=-\frac{L}{E_{x}} \frac{1}{\left(\frac{\varepsilon \zeta_{\mathrm{w}}}{\eta}-v z F\right)}$,

where $\varepsilon$ and $\eta$ are, respectively, the permittivity and viscosity of the electrolyte medium, $F$ Faraday's constant, $z$ and $v$, respectively, the charge number and mobility of fluorescein, $E_{x}$ the amplitude of the applied electric field, and $L$ the distance of the detector from the injection point. For the charge number of fluorescein at the various $\mathrm{pH}$ levels, we use the results of Sjoback et al. (1995), while the values of $v$ are taken according to our previous findings (Driehorst et al. 2010). The results of this analysis are presented in Fig. 2, where we also compare experimental $\zeta$ values reported in the literature (solid black line, (Kirby and Hasselbrink 2004) a compendium from several sources) and theoretical values derived using the surface charge model (dashed yellow line), proposed by Behrens et al. for silica surfaces (Behrens and Grier 2001). In this latter model, briefly, the $\zeta$-potential is found solving simultaneously the chemical equilibrium equation:

$\zeta(\sigma)=\frac{k T}{e} \ln \left(\frac{-\sigma}{e \Gamma+\sigma}\right)+\frac{\ln (10)}{e / k T}(p K-p H)-\frac{\sigma}{C^{\prime}}$

and Grahame's equation:

$\sigma(\zeta)=\frac{2 \varepsilon k T \kappa}{e} \sinh \left(\frac{e \zeta}{2 \kappa T}\right)$.

This is done in the limit of thin EDL. Here, $\sigma$ is the surface charge, $k$ Boltzmann's constant, $T$ the absolute temperature, $e$ the unit charge, $\kappa^{-1}$ the Debye length, $\Gamma$ the surface density of chargeable sites $\left(8 \mathrm{~nm}^{-2}\right), C$ Stern's capacitance $\left(0.3-2.9 \mathrm{~F} / \mathrm{m}^{2}\right)$ and $\mathrm{p} K$ the dissociation constant of $\mathrm{SiOH}$ (6-8.5).

Figure $2 \mathrm{a}$ presents the results of $\zeta$-potential measurements for $10 \mathrm{mM}$ buffer concentrations. Our values of $\zeta$ are in very good agreement with each other and show no dependence on channel type (i.e., manufacturer), implying that we do not have to account for effects related to different material properties. Additionally, we find a fairly good agreement with the data presented in (Kirby and Hasselbrink 2004) (solid black line), with our values shifted down by approximately $20-30 \mathrm{mV}$. While there are several possible reasons for this discrepancy, the systematic shift seems to point to a difference in counterion type in our experiments and/or temperature as the likely source. As for the surface charge model (dashed yellow line), we find a fair qualitative agreement by taking the values of $C=1 \mathrm{~F} / \mathrm{m}^{2}$ (typical value $C=2.9 \mathrm{~F} / \mathrm{m}^{2}$ ) and $\mathrm{p} K=6$ (typical value $\mathrm{p} K=7.5$ ).

Figure $2 \mathrm{~b}$ presents the results for the case of $1 \mathrm{mM}$ buffer concentration. The $\zeta$ potential derived from our measurements shows a dependence on channel size, except at $\mathrm{pH}$ 7. For $20 \mu \mathrm{m}$ channels, the results are in good agreement with data presented in the literature (black solid line) and show approximately the same amplitude shift already noticed for $10 \mathrm{mM}$ data. The agreement with the values predicted by the SCM is also fairly good. On the contrary, data for $1 \mu \mathrm{m}$ and $250 \mathrm{~nm}$ channels shows an unexpected behavior. Assuming that the charge of fluorescein is the same in the three channels, for $250 \mathrm{~nm}$ channels at $\mathrm{pH} 5$ we measure an increased electroosmotic flow, which in Fig. $2 \mathrm{~b}$ we attribute to a larger value of $\zeta$. According to (Bottenus et al. 2009), it is possible that at lower ionic strengths there are $\mathrm{pH}$ shifts within nanochannels. These would result in the fluorescein having a lower charge and therefore would affect our estimate of $\zeta$. However, if we attribute the change in $\zeta$ to a $\mathrm{pH}$ shift, we would have to assume a shift of $4 \mathrm{pH}$ units to reproduce the data at $20 \mu \mathrm{m}$, which does not seem to be a reasonable assumption. Another reason for the discrepancy may be the inadequacy of the models we are using to represent the physics within the channel. Recently, a newer "triplelayer" model (Wang and Revil 2010) has been proposed, which describes the addition of a metal ion adsorption layer that can also change the zeta potential and surface charge. Andersen et al. (2010) have also been developing a model, corroborated by experiments within nanochannels, that
Fig. 2 Zeta potential measured in the various channels using charged dyes and comparison with values derived from literature values (Kirby and Hasselbrink 2004) and surface charge model (van der Heyden et al. 2005)
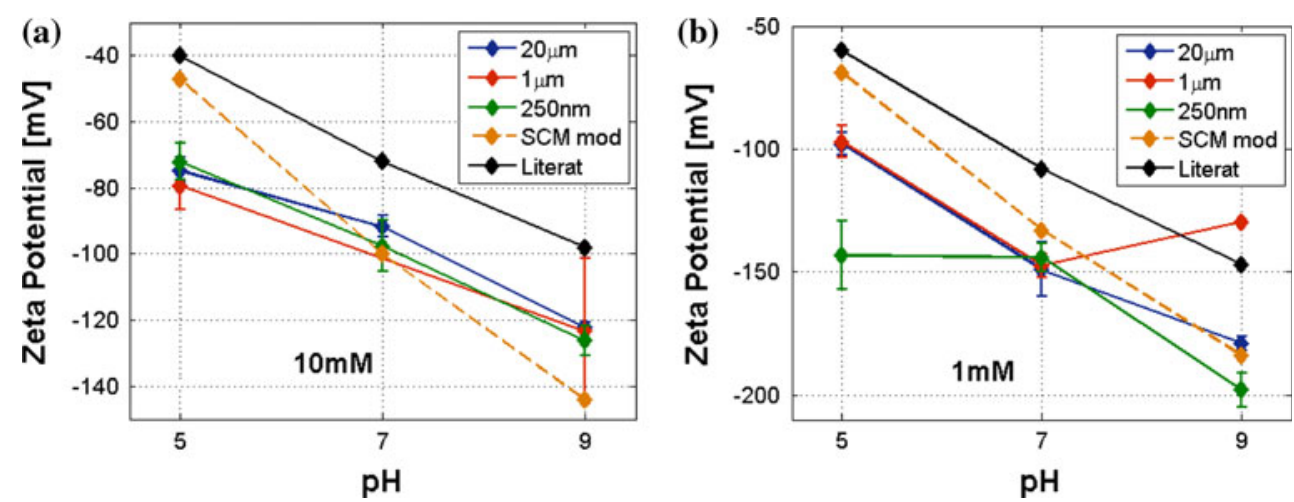
takes into account the chemistry at the surface, including the two different $\mathrm{p} K_{\mathrm{a}}$ values of a silica surface and the number of total surface charge sites. This surface chemistry is combined with the triple-layer model, the chemical equilibrium model, and the Poisson-Boltzmann equations for the bulk. In this model, the authors show that both the $\mathrm{p} K_{\mathrm{a}}$ value of the surface and the Stern layer capacitance can change with concentration. This fact may very well explain the discrepancy of zeta potential value with concentration, which is not accounted for in the models with respect to the results plotted above. We plan to further analyze this data with respect to the proposed model by Andersen in the near future.

A dependence on channel size is noticed also at $\mathrm{pH} 9$, where the variation in electroosmotic flow in $1 \mu \mathrm{m}$ and $250 \mathrm{~nm}$ channels is in the opposite direction. At this time, we do not know the origin of these changes in flow rate, and we assign them to a different "effective" $\zeta$.

In the remainder of this article, for each buffer concentration and $\mathrm{pH}$, we have used the average $\zeta$ values reported in this section, to derive the electrophoretic mobility of the particles from the data measured at that buffer condition.

\section{Electrokinetic separation data}

The results of our electrokinetic injection and separation experiments are summarized in Fig. 3, which shows the measured dependence of electrophoretic mobility on buffer type and concentration, for all channel geometries ( $\mathbf{\square}$ $20 \mu \mathrm{m}$ deep, $\diamond-1 \mu \mathrm{m}$ deep, $\bigcirc-250 \mathrm{~nm}$ deep). In this figure, yellow and green symbols refer to $50 \mathrm{~nm}$ particle data, while red and blue depict data for $100 \mathrm{~nm}$ particles (denoted also by larger markers, see Fig. 3a for an explanation of the symbols). Also, $10 \mathrm{mM}$ buffer data corresponds to yellow and red markers, while green and blue describe $1 \mathrm{mM}$ buffers. As stated above, for each combination of experimental conditions $(\mathrm{pH}$, ionic strength, electric field, channel type, observation points), the measurements were repeated three times. Electrophoretic mobility, $\mu_{\mathrm{EP}}$ was obtained by subtracting electroosmotic velocity from the measured data, $v_{\mathrm{PART}}$ and dividing by the applied electric field,

$\mu_{\mathrm{EP}}=\frac{\nu_{\mathrm{EP}}}{E_{x}}=\frac{\nu_{\mathrm{PART}}}{E_{x}}-\frac{\varepsilon \zeta_{\mathrm{W}}}{\eta}$

using for $\zeta_{\mathrm{w}}$ the values obtained from our measurements and presented in the previous section. Note that this formula does not take into account EDL effects on the electroosmotic flow, which we consider negligible in this case, since for 10 and $1 \mathrm{mM}$ buffer concentrations the Debye length amounts to, respectively, 3 and $10 \mathrm{~nm}$, and channel heights were 25 to 20,000 times larger at $250 \mathrm{~nm}, 1 \mu \mathrm{m}$, and $20 \mu \mathrm{m}$.

For each given channel type, there are at least two trends that are apparent analyzing the data in Fig. 3. The first one is the decrease of mobility (in absolute value) as the $\mathrm{pH}$
Fig. 3 Electrophoretic mobility of $50 \mathrm{~nm}$ (yellow, green) and $100 \mathrm{~nm}$ (red and blue and larger marker size) nanoparticles as a function of $\mathrm{pH}$ and buffer concentration: $10 \mathrm{mM}$ (yellow and red) and $1 \mathrm{mM}$ (green and blue). a data collected in $20 \mu \mathrm{m}$ deep channels ( $\mathbf{\square})$; b same as (a), but including $1 \mu \mathrm{m}$ deep channels $(\diamond)$; c same as $(\mathbf{b})$, but including data for $250 \mathrm{~nm}$ channels $(\bigcirc)$. Symbols represent average data (3 electric fields, $>1$ observation points), while the error bar is one standard deviation. (Color figure online)
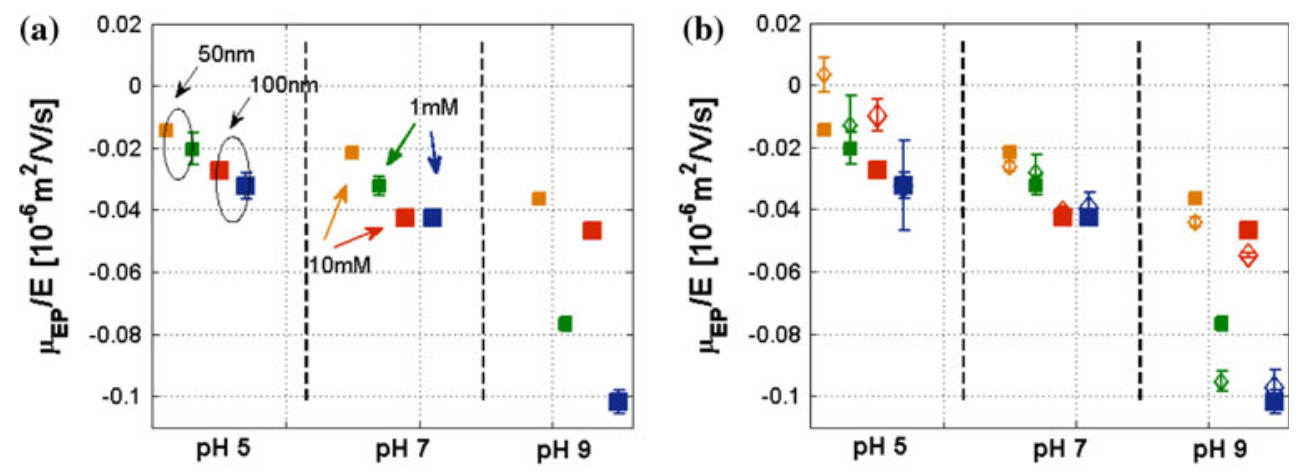
decreases. We attribute this general trend to a change in the effective charge of the particles: as the background electrolyte becomes more acidic, protonation of the carboxyl groups results in an overall lower effective charge of the particles and, hence, in a lower electrophoretic velocity. In particular, our measurements indicate that the isoelectric point (IEP) of these particles is around $\mathrm{pH} 4$, in good agreement with the reported values of $\mathrm{p} K_{\mathrm{a}}=8$ for carboxylic acids on a surface (Wang et al. 1992). A similar trend can be noticed also as the buffer molarity decreases from 10 to $1 \mathrm{mM}$ : for each buffer type, at $1 \mathrm{mM}$ concentration the particles have a larger (in magnitude) electrophoretic mobility. This effect can be explained by an increased EDL around the particles, which results in a larger effective $\zeta$-potential, and therefore a larger electrophoretic velocity.

However, the most interesting behavior in terms of mobility variation can be observed as a function of channel size. More precisely, from Fig. 3b, we notice that, while for phosphate buffer $(\mathrm{pH} 7)$ the data collapses quite well for both height channels ( 20 and $1 \mu \mathrm{m}$ ), for acetate and borate there is an opposite trend: the absolute value of mobility decreases for acetate (pH 5) with decreasing height, while for borate $(\mathrm{pH} \mathrm{9)}$ the mobility increases. This fact cannot be explained by changes in effective charge, since protonation effects should not depend on channel size at this scale. Also, double layer induced phenomena (i.e., nonuniform flow profile coupled to transverse electric field as illustrated in Pennathur and Santiago (2005a)), cannot be invoked to explain the data, since we have a very thin EDL $\left(\lambda_{\mathrm{D}} \leq 10 \mathrm{~nm}\right.$ vs. depths of 1 and $\left.20 \mu \mathrm{m}\right)$. Possible adsorption effects are independent of channel size and therefore also not able to justify these results.

Instead, we interpret this trend as being due to the hydrodynamic and electrokinetic effects, which start to play a role when the unbounded domain limit is violated (e.g., particle size is not small compared to the size of the channel). There have been many studies concerning corrections, which arise in this setting (Keh and Chiou 1996; Yariv and Brenner 2002; Ye et al. 2002; Yariv and Brenner 2003a, b; Ye and Li 2004). In general, the equations describing the coupled electrokinetic and hydrodynamic coupling are nonlinear and difficult to handle analytically, often requiring solution by advanced CFD numerical methods. A full treatment of this problem is beyond the intended scope of this paper. Instead, we discuss our hypotheses on a mechanistic level and we defer to future work the development of a more theoretical study of the reported experimentally observed phenomena.

As a brief summary of the main results that we report for the case of spherical particles, it has been found that particle velocity is determined by the balance of two opposing effects: viscous retardation near a non-conducting boundary and enhanced electric-field as the particle-wall separation becomes smaller. The net effect is that particle velocity is expected to decrease as the channel size becomes gradually smaller, until electric field effects become dominant, inducing a larger traveling velocity. These predictions are consistent with the single particle experimental studies of Xuan et al. (2005) and Xuan et al. (2006).

With regard to our results, we posit that the lower charge of wall surface and particles in $10 \mathrm{mM}$ acetate buffer $(\mathrm{pH}$ 5) allows the particles to get closer to the wall. The result is that the local higher electric field enhances the particles velocity (by $118 \%$ for $50 \mathrm{~nm}$ particles and $62 \%$ for $100 \mathrm{~nm}$ ). At $1 \mathrm{mM}$, the larger charge and Debye length create a force that tends to push the particles further away from the wall, which not only increases the contribution of viscous drag to the final velocity, but also slows down the particles less than in the $10 \mathrm{mM}$ case (since there is only $34 \%$ increase for $50 \mathrm{~nm}$ particles and practically no variation for $100 \mathrm{~nm}$ ). At $\mathrm{pH} 9$, viscous drag appears to be the predominant force, resulting in a velocity that decreases both with channel size and buffer concentration (by -22 to $-25 \%$ for $50 \mathrm{~nm}$ particles, in 10 and $1 \mathrm{mM}$, respectively, and -16 to approximately $4 \%$ for $100 \mathrm{~nm}$ particles, also in $10 \mathrm{mM}$ and $1 \mathrm{mM}$, respectively), as expected from theoretical predictions (Keh and Chen 1988; Yariv and Brenner 2003a; Ye et al. 2005).

Finally, in Fig. 3c data for nanochannels is shown in which it is seen that a further reduction in channel size results in the expected increase in velocity of the negatively charged polystyrene spheres. This becomes particularly significant at $1 \mathrm{mM}$. We hypothesize this is a consequence of the predominant contribution of the enhanced electric fields within the nanochannels. Table 1 shows the percentage change in mobility of these particles between $20 \mu \mathrm{m}$ and $250 \mathrm{~nm}$ deep channels. Although substantial, this change is far less than the increase predicted by (Keh and Anderson 1985) for slit channels, and which should amount approximately to the ratio of the two channel depths. We presume that this discrepancy is due to the relative large Debye length to particle radius ratio, which in (Keh and Anderson 1985) is assumed to be very small.

Examining data collected within the $250 \mathrm{~nm}$ channel in more detail (Fig. 3c), we find that the velocity for $100 \mathrm{~nm}$ particles is so increased at $1 \mathrm{mM}$ buffer concentrations that in some cases there is almost no separation between the two particle plugs. This effect is shown in more detail in Fig. 4, where one can see that, in nanochannels and at $1 \mathrm{mM}$, the difference in mobility is greatly reduced from the case of a microchannel. This result is in contrast to what found in Xuan et al. (2006). Here, the authors study the electrophoretic motion of single spherical polystyrene 
Table 1 Electrophoretic mobility change in $1 \mu \mathrm{m}$ and $250 \mathrm{~nm}$ channels versus $20 \mu \mathrm{m}$ channels. For this latter case, notice the sharper increase at lower buffer concentration

\begin{tabular}{|c|c|c|c|c|c|}
\hline & & \multicolumn{2}{|c|}{$\Delta \mu_{\mathrm{EP}}$ from $20 \mu \mathrm{m}$ to $1 \mu \mathrm{m}$} & \multicolumn{2}{|c|}{$\Delta \mu_{\mathrm{EP}}$ from $20 \mu \mathrm{m}$ to $250 \mathrm{~nm}$} \\
\hline & & $10 \mathrm{mM}$ & $1 \mathrm{mM}$ & $10 \mathrm{mM}$ & $1 \mathrm{mM}$ \\
\hline \multirow[t]{2}{*}{ Acetate (pH 5) } & $50 \mathrm{~nm}$ & +118 & +34 & +100 & +37 \\
\hline & $100 \mathrm{~nm}$ & +62 & 0 & +31 & +42 \\
\hline \multirow[t]{2}{*}{ Phosphate (pH 7) } & $50 \mathrm{~nm}$ & $-15^{\mathrm{a}}$ & $+9^{\mathrm{a}}$ & -19 & +59 \\
\hline & $100 \mathrm{~nm}$ & $+1^{\mathrm{a}}$ & $+4^{\mathrm{a}}$ & -2 & +54 \\
\hline \multirow[t]{2}{*}{ Borate (pH 9) } & $50 \mathrm{~nm}$ & -22 & -25 & +24 & +33 \\
\hline & $100 \mathrm{~nm}$ & -16 & +4 & +35 & +49 \\
\hline
\end{tabular}

${ }^{a}$ Although strictly not zero, the variation in mobility is practically within the error bars for these entries
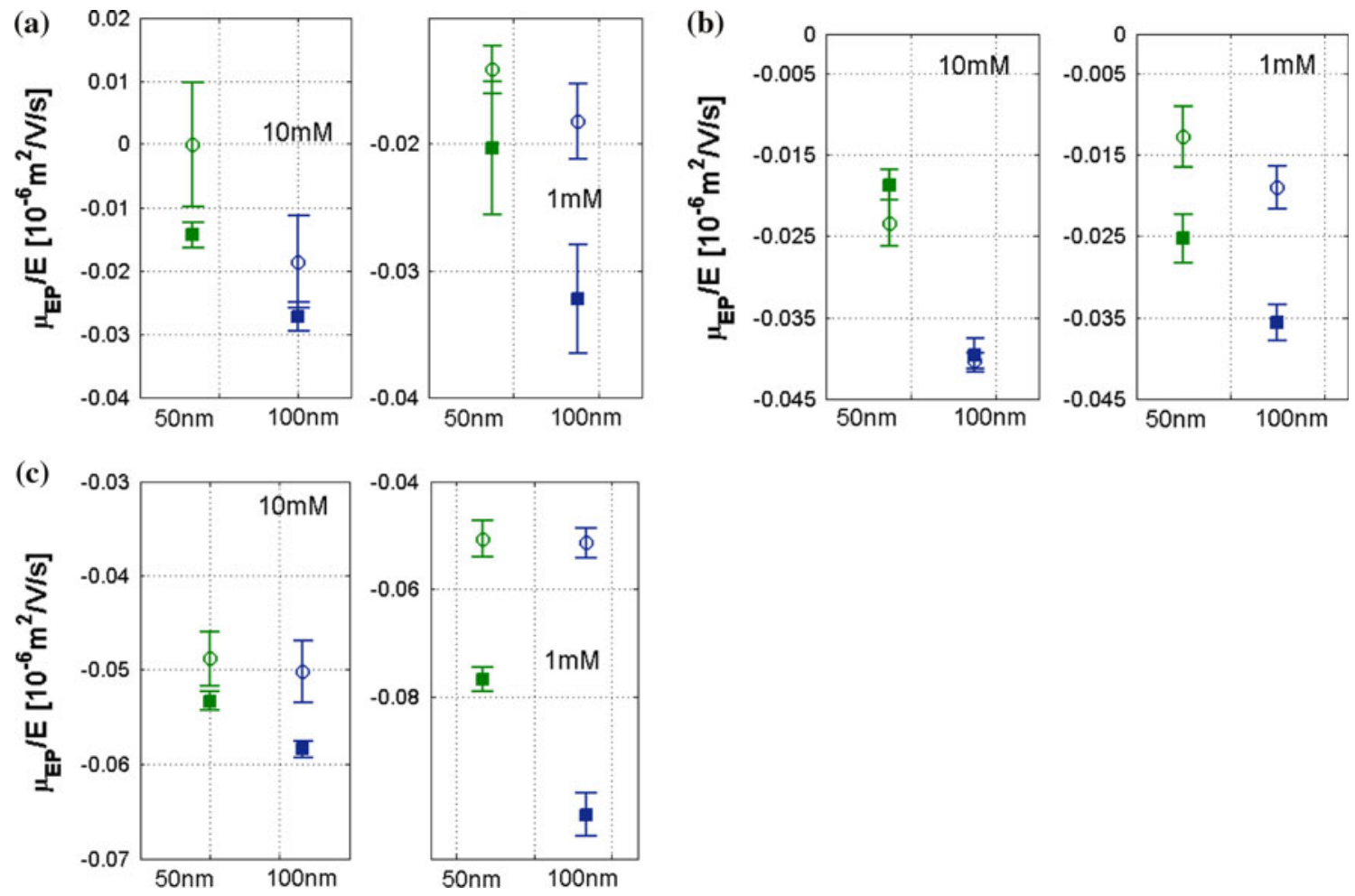

Fig. 4 Comparison of electrophoretic mobility of spherical nanoparticles measured in microchannels (closed square) and nanochannels (open circle) for a acetate, $\mathbf{b}$ phosphate, and $\mathbf{c}$ borate buffer

particles in rectangular PDMS channels with varying cross section. By measuring their velocity at the entrance and within the channel neck, it is shown that larger particles are less accelerated than smaller ones. The effect is attributed to viscous retardation from the channel sidewalls. Our results do not agree with these findings (see Table 1: in $250 \mathrm{~nm}$ channels $-1 \mathrm{mM}$ buffers, $100 \mathrm{~nm}$ particles are more accelerated than $50 \mathrm{~nm}$ particles). For the nanochannels, one possible explanation is that the repulsion between the negatively charged particles and the negatively charged channel walls plays a significant role. As a consequence of the length scale of the repulsive interactions and the channel dimensions, such repulsion could play a role in pushing particles more toward the center of the channel. This relative increase in the concentration of particles toward the channel center could reduce the relative viscous retardation due to proximity to the sidewalls. We note here that although such effects possibly could be used to tune separation performance, in this instance, we see a degradation of separation efficacy, resulting in lower resolution. The designer should be aware of this potential issue and be prepared to compensate for it in chip designs for separations which rely on nanoscale features of the device. 


\section{Effective coefficient for the diffusion and dispersion of the concentration profile}

An effective diffusion coefficient, $D$, can be defined to characterize how an initial spatial distribution of particles spreads out over time. This is done by using for each time a Gaussian fit to the concentration profile in space. More precisely, for an initial concentration profile tightly localized in space, the diffusion coefficient $D$ characterizes how the variance $\sigma^{2}(t)$ of the Gaussian distribution increases in time $t$ :

$\sigma^{2}(t)=2 D t$.

For particles undergoing purely Brownian motion, the value of the diffusion coefficient $D$ is given by the StokesEinstein relation:

$D=\frac{k T}{\gamma}$,

where $k$ is Boltzmann's constant, $T$ the absolute temperature, and $\gamma$ the hydrodynamic drag coefficient of a particle.

It should be mentioned that the Stokes-Einstein relation relies upon the fluctuation-dissipation principle (FLDP) of statistical mechanics for a system near thermodynamic equilibrium (Reichl 1998). We caution that in the current setting, where the particles are subject to external fields and, possibly, to complicated electrokinetic effects, the assumptions underlying the use of FLDP could be violated. For our present purposes Eq. 2 will be used primarily as a base-line for comparison of the spread observed in experiments with what would be expected if particles were subject purely to Brownian motion. Throughout the subsequent discussion, it should be understood that a more sophisticated theory for the diffusivity of particles may be required, if the electrokinetic effects induced by the external fields are found to result in a significant violation of FLDP.

In Eq. 2, a drag coefficient is required, which in practice could depend on many factors. These factors include the particle proximity to the channel walls or even the particular nanoparticle considered, since the population of nanoparticles is expected to exhibit some variations from features of the manufacturing process. For our present purposes, we shall take $\gamma$ to be a representative value for the drag coefficient and use in our base-line calculations the Stokes drag for a spherical particle:

$\gamma=6 \pi \eta r$

where $\eta$ is the fluid viscosity and $r$ is the radius of the particle.

For the smaller particles used in the experiments, Eqs. 2 and 3 yield a base-line diffusivity of $D^{\prime}=8.55 \mathrm{e}^{-12}$, and for the larger particles a base-line diffusivity of
Table 2 Experimentally measured diffusion coefficients in $20 \mu \mathrm{m}$ channels from Gaussian fits of intensity profiles at the three detection points

\begin{tabular}{lllll}
\hline & & \multicolumn{3}{l}{ Measured diffusion coefficient } \\
\cline { 3 - 5 } & & $5 \mathrm{~mm}$ & $10 \mathrm{~mm}$ & $20 \mathrm{~mm}$ \\
\hline Acetate & $50 \mathrm{~nm}$ & $1.54 \mathrm{E}-09$ & $3.23 \mathrm{E}-09$ & $8.29 \mathrm{E}-10$ \\
& $100 \mathrm{~nm}$ & $2.47 \mathrm{E}-08$ & $5.53 \mathrm{E}-08$ & $1.70 \mathrm{E}-08$ \\
Phosphate & $50 \mathrm{~nm}$ & $3.80 \mathrm{E}-09$ & $8.80 \mathrm{E}-09$ & $1.20 \mathrm{E}-08$ \\
& $100 \mathrm{~nm}$ & $1.70 \mathrm{E}-09$ & $2.20 \mathrm{E}-09$ & $1.20 \mathrm{E}-08$ \\
Borate & $50 \mathrm{~nm}$ & $2.50 \mathrm{E}-09$ & $5.00 \mathrm{E}-09$ & $9.70 \mathrm{E}-09$ \\
& $100 \mathrm{~nm}$ & $1.50 \mathrm{E}-09$ & $4.50 \mathrm{E}-09$ & $9.30 \mathrm{E}-09$ \\
\hline
\end{tabular}

$D^{\prime}=4.37 \mathrm{e}^{-12}$. In Table 2, we report values for the effective diffusion coefficient $D$ obtained from experimental measurements, using a Gaussian fit to the concentration profile over time and Eq. 1. It is found that the effective diffusion coefficient $D$ is manifestly much larger (two orders of magnitude) than the diffusivity expected purely from Brownian motion of the nanoparticles. This indicates that effects other than pure diffusion are responsible for the spread observed in the concentration profile of the collection of particles.

To simplify our analysis, we shall consider only the data obtained from the $20 \mu \mathrm{m}$ channels at $10 \mathrm{mM}$ buffer concentrations. This corresponds to the case in which the EDL is expected to be thin $\left(\lambda_{\mathrm{D}}=3 \mathrm{~nm}\right)$ and the dimensions of the fluid domain within the channel are expected to be significantly larger than the particle radius, allowing for treatment approximately as an unbounded domain. For this channel geometry, we have collected data at three detection points: 5,10 , and $20 \mathrm{~mm}$. This can also be regarded as taking measurements at different time instants in the evolution of the concentration profile, as the plug travels along the injection channel. Data collected at each of these measurement points is fit to a Gaussian and results in an estimate of $D$ (see Sect. 2 for details on the image processing used).

From Table 2, one notices that: (a) the experimental estimates of the effective coefficient $D$ is much larger than that expected from pure diffusion, and that (b) except for acetate, the values of $D$ actually increase over time (i.e., as the observation distance increases from the injection point). We will discuss the case of acetate separately.

While there are many possible electrokinetic effects that could be considered, one basic effect, which could account for the observed effective coefficient $D$, is that the population of nanoparticles is heterogeneous, as a consequence of the manufacturing process. This fact would be qualitatively consistent with the increase of $D$ over time: if we have sub-populations of particles with different zeta, they will tend to progressively separate into distinct plugs, as 
time passes. In fact, according to theory (Henry 1931; Ottewill and Shaw 1972; Hunter 1981; Hunter 1994; Probstein 1994; Kirby 2009), under the influence of an applied electric field $E_{x}$, a spherical particle moves through an electrolyte solution with an electrophoretic velocity, $v_{\mathrm{EP}}$, given by the following expression:

$v_{\mathrm{EP}}=-f\left(\zeta_{\mathrm{p}}, r, \lambda_{\mathrm{D}}\right) \frac{\varepsilon \zeta_{\mathrm{P}}}{\eta} E_{x}$,

where $\varepsilon$ and $\eta$ are, respectively, the permittivity and viscosity of the medium, and $\zeta_{\mathrm{p}}$ is the $\zeta$-potential of the particle. The multiplicative factor $f\left(\zeta_{\mathrm{p}}, r, \lambda_{\mathrm{D}}\right)$ is known as Henry's function and describes the effect of finite double layers on particle velocity. More precisely, if the Debye length $\lambda_{\mathrm{D}}$ is not thin compared to particle size, the applied electric field cannot be considered uniform within the double layer. The net effect is a decrease $(f \leq 1)$ of particle velocity, by an amount that depends non-linearly on $\zeta_{\mathrm{p}}$ and the ratio between the radius of the particle, $r$, and $\lambda_{D}$ (Ottewill and Shaw 1972).

One could object that if particles of similar size have different $\zeta$, their mutual interactions may affect their respective electrophoretic mobility in a way that is not described by Eq. 4. This problem has been studied analytically in Chen and Keh (1988), where it is shown that the strength of the interaction between two non-conducting spherical particles depends on the ratio between their $\zeta$ values, their separation distance and their relative position with respect to the applied electric-field. The authors also demonstrate that for particle collections, the interaction is weighted by the relative volume fraction. Although we do not have precise quantitative information about volume fractions for each particle sub-population, the maximum possible value for each is expected to be rather small due to the dilute suspension, $\mathrm{O}\left(10^{-5}\right)$. In our case, this suggests that such collective effects contribute only negligibly to the electrophoretic velocity.

Another basic effect we have considered, besides heterogeneity in the zeta potential of the nanoparticles, is an increase in the estimated diffusion coefficient caused by reversible adsorption (Bello et al. 1995). However, we do not see the typical tailed (triangle-like) peaks that are commonly found to accompany this phenomenon (data not shown). This has led us to believe that heterogeneity might instead be the most likely explanation.

Our hypothesis concerning the measured value of $D$ could in principle be tested if we knew in advance the $\zeta$-potential values of the population and if the $\zeta$-potential describes reliably the mobility of each of the sub-populations of particles, as in Eq. 4. Unfortunately, the $\zeta$-potential (or surface charge) value for nanoparticles used in this study is not characterized by the manufacturer and is, in general, hard to determine with confidence using currently available devices.
Variations in the number of attached carboxyl groups within the same particle populations are not considered unlikely, as a result of different amounts of initiator and adsorbed surfactant molecules involved in the manufacturing process (from private communication). As a consequence, the particles could have a range of zeta potential values. Our measurements using a ZetasizerNano (Malvern) suggest a rather wide range of values (Table 3), however, in many cases the values obtained were not compatible with our electrophoretic mobility measurements. We believe that this fact is due to intrinsic limitations of the instrument in analyzing mixtures of particles with $\zeta$ values very close to each other. Therefore, although they are in line with our hypothesis, we do not feel very confident in the ZetasizerNano results.

Instead, to show the plausibility of our hypothesis we shall explore whether our electrophoretic data set and $D$ could be obtained from a heterogeneous population of nanoparticles for a plausible range of effective $\zeta$ values and physical parameters. For the physical parameters of the experiment, a failure to find a plausible range of $\zeta$ would invalidate our hypothesis.

To model the experiments, the concentration profile $c(x$, $t$ ) of the heterogeneous population is defined in terms of the concentration profiles $c_{k}(x, t)$ of each sub-population:

$c(x, t)=\sum_{k} c_{k}(x, t)$.

The concentration profile of each sub-population is modeled by the solution of the one-dimensional transport equation:

$\frac{\partial c_{k}}{\partial t}=-v_{k} \frac{\partial c_{k}}{\partial x}+D_{k} \frac{\partial^{2} c_{k}}{\partial x^{2}}, \quad c(x, 0)=w_{k} \phi(x)$.

The $v_{k}$ is the velocity, $D_{k}$ the Brownian diffusion coefficient of the $k$ th nanoparticle sub-population, $\Phi(x)$ the initial plug concentration profile, $w_{k}$ the concentration of the nanoparticle sub-population. Throughout, the $D_{k}$ will be taken to be the same for each sub-population, since the particles are quite uniform in size (according to our own measurements and data provided by Polysciences). The primary source of heterogeneity we consider is in the electrophoretic velocity of the particles, arising from variability in the zeta potential values.

For the $20 \mu \mathrm{m}$ channel and for the buffer concentrations considered, we invoke the thin EDL approximation. This allows for the electrophoretic velocity $v_{k}$ to be expressed by the Helmholtz-Smoluchowski relation:

$v=\frac{\varepsilon}{\eta}\left(\zeta_{\mathrm{w}}-\zeta_{\mathrm{p}, \mathrm{eff}}\right) E_{x}$

where $\zeta_{\mathrm{w}}$ is the wall $\zeta$-potential (from Sect. 2.4) and $\zeta_{\mathrm{p} \text {,eff }}=f\left(\zeta_{\mathrm{p}}, r, \lambda_{\mathrm{D}}\right) \zeta_{\mathrm{p}}$ is the effective $\zeta$-potential of the particle, including Henry's correction factor (Eq. 4). If we suppose that each initial particle collection (50 and $100 \mathrm{~nm}$ 
Table 3 Comparison of $\zeta$-potential values measured by Zetasizer and estimated by us

\begin{tabular}{llllllll}
\hline$\zeta_{\mathrm{p}, \text { eff }}(\mathrm{mV})$ & $10 \mathrm{mM}$ Borate & & & \multicolumn{1}{l}{$10 \mathrm{mM}$ Phosphate } & & \multicolumn{2}{c}{$10 \mathrm{mM}$ Acetate } \\
\cline { 2 - 3 } & Our estim & Malvern & & Our estim & Malvern & & Our estim \\
\hline $50 \mathrm{~nm}$ & {$[-55,-40]$} & {$[-100,-35]$} & & {$[-42,-25]$} & {$[-125,-25]$} & {$[-20,-10]$} & {$[-105,-25]$} \\
$100 \mathrm{~nm}$ & {$[-60,-50]$} & {$[-95,-30]$} & & {$[-55,-45]$} & {$[-105,-45]$} & {$[-45,-20]$} & {$[-105,-35]$} \\
\hline
\end{tabular}

particles) in the sample plug is composed of subsets corresponding to a distribution of $\zeta$-potential values, each subset will travel at a slightly different velocity, giving rise to an ensemble of plugs slightly shifted from each other at the detection point. The amplitude of these plugs will depend on the size of the corresponding sub-population. Throughout our fitting, we assume the concentration of sub-populations with different $\zeta$-potential values to follow a Gaussian distribution, with mean corresponding to the average $\zeta$-potential value of the heterogeneous population and with two standard deviations corresponding to the range of $\zeta$-potential values reported. We obtain the mean and range of $\zeta$-potential values of the sub-populations from the experimental data set. This is done by varying the $\zeta$ potential values to fit the concentration profiles over time produced by Eq. 5 to the Gaussians with the reported $D$ coefficients in Table 2.

This procedure for the case of $100 \mathrm{~nm}$ particles is shown in Fig. 5. Here, we have reproduced the concentration profile at the three experimental observation points: 5, 10, and $20 \mathrm{~mm}$. The thick solid lines represent the Gaussian fits obtained taking $\zeta=-55 \mathrm{mV}$ (average of the range considered, see Table 3 ) and $D$ equal to the measured

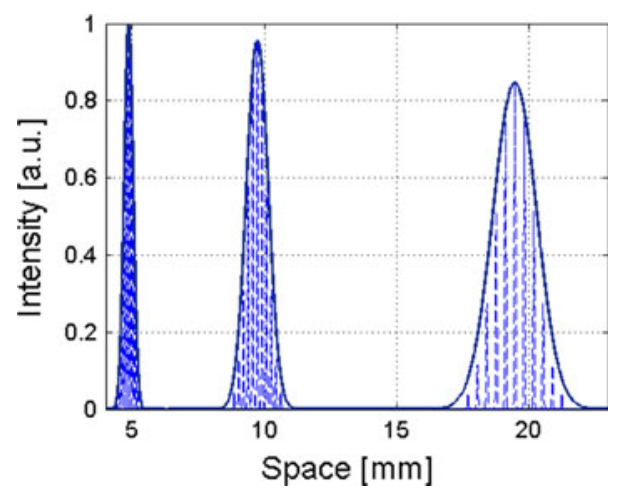

Fig. 5 Illustration of the method used to determine a plausible range for the effective $\zeta$-potential of a heterogeneous population of nanoparticles. The concentration profile of Eq. 5 is fit to Gaussians with the measured diffusion coefficients $D$ reported in Table 2 . The specific case illustrated here corresponds to $100 \mathrm{~nm}$ particles in a borate buffer. The concentration profiles of the sub-populations are shown as (light, dashed lines). The fit of the total concentration profile of the population to the Gaussian concentration profile for the experimentally measured values of $D$ are shown as (dark, solid lines). This fitting procedure determines a range of $\zeta$-potential values for the heterogeneous population of nanoparticles values at those observation points. The dashed lines represent the distributions of the single sub-groups of particles having the same $\zeta$, within the range considered ([-60 $50] \mathrm{mV}$ in this example).

We have found that our measured diffusion coefficients, $D_{\text {meas }}$, at all observation points, are consistent with the range of $\zeta$ values reported in Table 3 , which summarizes our estimated $\zeta$-potentials and compares them with the values obtained from a ZetasizerNano (Malvern). It should be mentioned that the duration of the plugs obtained by our fitting procedure also matches the measured values. The only exception is the data regarding acetate buffer at $20 \mathrm{~mm}$. In the case of acetate, particles adsorb noticeably to the walls, in agreement with (Visser 1976), who found that particle adhesion is maximum at the point of zero charge. For the case of the acetate buffer, we hypothesize that at $20 \mathrm{~mm}$ the particles with the lowest charge have disappeared from the plugs, which manifests in the data by narrower Gaussians (and hence in lower values of $D$ ). In the absence of adsorption, the method we have outlined appears to provide a novel way to estimate a range for the $\zeta$-potential values of a heterogeneous population of nanoparticles.

\section{Concluding Remarks}

In this article, we reported results of our experimental investigation on the transport and separation behavior of 50 and $100 \mathrm{~nm}$ spherical polystyrene nanoparticles confined in $20 \mu \mathrm{m}, 1 \mu \mathrm{m}$, and $250 \mathrm{~nm}$ deep fluidic channels. We have found that mobility of the particles depends on coupled effects induced by changes in $\mathrm{pH}$, ionic strength, and channel height. In particular, we have shown that, depending on $\mathrm{pH}$, the variation of particle mobility can show opposite behavior than expected upon reducing the size of the microchannel. In addition, we have shown that there is a large increase in mobility in nanoscale channels, which is further enhanced for decreasing electrolyte ionic strength. This effect could be utilized to tune separation performance, but also has the potential to degrade it, as shown in our results. Finally, we have used the diffusivity measurements to determine the range of effective zeta potentials of our spherical particles. Our estimates are compatible with the measured electrophoretic mobility and 
the method could prove useful in the case of heterogeneous populations of particles, which exhibit a distribution of zeta potential values.

Acknowledgments The authors would like to thank Dr. R. White for useful discussions during the preparation of the manuscript.

Open Access This article is distributed under the terms of the Creative Commons Attribution Noncommercial License which permits any noncommercial use, distribution, and reproduction in any medium, provided the original author(s) and source are credited.

\section{References}

Andersen MB, Frey $\mathbf{J}$ et al (2010) Investigation of the solid/liquid interface of coated silica nanochannels during capillary filling. In: 9th ELKIIN Conf., Finland. Accepted 6-10 June 2010

Behrens SH, Grier DG (2001) The charge of glass and silica surfaces. J Chem Phys 115(14):6716-6721

Bello MS, Zhukov MY et al (1995) Combined effects of nonlinear electrophoresis and nonlinear chromatography on concentration profiles in capillary electrophoresis. J Chromatogr A 693(1):113-130

Benke M, Shapiro E et al (2008) An efficient multi-scale modelling approach for ssDNA motion in fluid flow. J Bionic Eng 5(4):299-307

Bharadwaj R, Santiago JG et al (2002) Design and optimization of onchip capillary electrophoresis. Electrophoresis 23(16):27292744

Bottenus D, Oh YJ et al (2009) Experimentally and theoretically observed native $\mathrm{pH}$ shifts in a nanochannel array. Lab Chip 9(2):219-231

Caslavska J, Thormann W (2001) Electrophoretic separations in PMMA capillaries with uniform and discontinuous buffers. J Microcolumn Sep 13(2):69-83

Chen SB, Keh HJ (1988) Electrophoresis in a dilute dispersion of colloidal spheres. Aiche J 34(7):1075-1085

Dickens JE, Gorse J et al (1994) Dependence of electroosmotic flow in capillary electrophoresis on group-I and group-II metal-ions. J Chromatogr B Biomed Appl 657(2):401-407

Driehorst T, O'Neill P et al (2010) Characterization of Agn:DNA nanoclusters by capillary electrophoresis in microfluidic channels (submitted to Langmuir)

Dukhin SS, Derjaguin BV (1974) Elektrokinetic phenomena. In: Matijevic E (eds) Surface and colloid science, vol 7. John Wiley Interscience, New York

Ennis J, Anderson JL (1997) Boundary effects on electrophoretic motion of spherical particles for thick double layers and low zeta potential. J Colloid Interface Sci 185(2):497-514

Ghosh S, Mashayekhi H et al (2008) Colloidal behavior of aluminum oxide nanoparticles as affected by $\mathrm{pH}$ and natural organic matter. Langmuir 24(21):12385-12391

Guzman KAD, Finnegan MP et al (2006) Influence of surface potential on aggregation and transport of titania nanoparticles. Environ Sci Technol 40(24):7688-7693

Henry DC (1931) The cataphoresis of suspended particles. Part I. The equation of cataphoresis. Proc R Soc Lond 133(821):106-129

Hsu JP, Kao CY (2002) Electrophoresis of a finite cylinder along the axis of a cylindrical pore. J Phys Chem B 106(41):10605-10609

Huckel E (1924) The cataphoresis of the sphere. Phys Zeitschrift 25:204-210
Hunter RJ (1981) Zeta potential in colloid science: principles and applications. Academic Press, New York

Hunter RJ (1994) Introduction to modern colloid science. Oxford University Press, Oxford

Keh HJ, Anderson JL (1985) Boundary effects on electrophoretic motion of colloidal spheres. J Fluid Mech 153(Apr):417-439

Keh HJ, Chen SB (1988) Electrophoresis of a colloidal sphere parallel to a dielectric plane. J Fluid Mech 194:377-390

Keh HJ, Chiou JY (1996) Electrophoresis of a colloidal sphere in a circular cylindrical pore. Aiche J 42(5):1397-1406

Kirby BJ (2009) Micro- and nanoscale fluid mechanics for engineers: transport in microfluidic devices. http://www.kirbyresearch.com/ index.cfm/wrap/textbook/microfluidicsnanofluidics.html

Kirby BJ, Hasselbrink EF (2004) Zeta potential of microfluidic substrates: 1. Theory, experimental techniques, and effects on separations. Electrophoresis 25(2):187-202

Kosmulski M, Matijevic E (1992) Zeta-potentials of silica in water alcohol mixtures. Langmuir 8(4):1060-1064

Kuo TC, Sloan LA et al (2001) Manipulating molecular transport through nanoporous membranes by control of electrokinetic flow: effect of surface charge density and Debye length. Langmuir 17(20):6298-6303

Liu H, Bau HH et al (2004) Electrophoresis of concentrically and eccentrically positioned cylindrical particles in a long tube. Langmuir 20(7):2628-2639

Majumder SR, Choudhury N et al (2007) Enhanced flow in smooth single-file channel. J Chem Phys 127(5):054706(1)-054706(5)

Maron SH, Bowler WW (1948) The electrophoretic mobility of typeIII Gr-S latex. J Am Chem Soc 70(11):3893-3896

Maron SH, Turnbull D et al (1948) The electrophoretic mobility of type-II Gr-S latex. J Am Chem Soc 70(2):582-587

Morrison FA (1970) Electrophoresis of a particle of arbitrary shape. J Colloid Interface Sci 34:210

Mosier BP (2001) Photobleached-fluorescence imaging of microflows. $\mathrm{PhD}$ Thesis, Stanford

Ottewill R, Shaw J (1972) Electrophoretic studies on polystyrene latices. J Electroanal Chem 37:133-142

Pennathur S, Santiago JG (2005a) Electrokinetic transport in nanochannels. 1. Theory. Anal Chem 77(21):6772-6781

Pennathur S, Santiago JG (2005b) Electrokinetic transport in nanochannels. 2. Experiments. Anal Chem 77(21):6782-6789

Probstein RF (1994) Physicochemical hydrodynamics. Wiley, New York

Qian SZ, Wang AH et al (2006) Electrophoretic motion of a spherical particle in a converging-diverging nanotube. J Colloid Interface Sci 303(2):579-592

Reichl LE (1998) A modern course in statistical physics. John Wiley and Sons

Scales PJ, Grieser F et al (1992) Electrokinetics of the silica solution interface-a flat-plate streaming potential study. Langmuir 8(3):965-974

Schoch RB, Renaud P (2005). Ion transport through nanoslits dominated by the effective surface charge. Appl Phys Lett 86(25):253111(1)-253111(3)

Schwer C, Kenndler E (1991) Electrophoresis in fused-silica capillaries - the influence of organic-solvents on the electroosmotic velocity and the zeta-potential. Anal Chem 63(17):1801-1807

Shugai AA, Carnie SL (1999) Electrophoretic motion of a spherical particle with a thick double layer in bounded flows. J Colloid Interface Sci 213(2):298-315

Sjoback R, Nygren J et al (1995) Absorption and fluorescence properties of fluorescein. Spectrochim Acta A Mol Biomol Spectrosc 51(6):L7-L21

Smoluchowski M (1921) Handbuch der Elektrizitaet und das Magnetismus. In: Graetz L (ed) vol 2. Barth, Leipzig, p 366 
van der Heyden FHJ, Stein D et al (2005) Streaming currents in a single nanofluidic channel. Phys Rev Lett 95(11):116104(1)116104(5)

Visser J (1976) The adhesion of colloidal polystyrene particles to cellophane as a function of $\mathrm{ph}$ and ionic strength. J Colloid Interface Sci 55(3):664-677

Wang J, Frostman LM et al (1992) Self-assembled thiol monolayers with carboxylic-acid functionality-measuring $\mathrm{pH}$-dependent phase-transitions with the quartz crystal microbalance. J Phys Chem 96(13):5224-5228

Wang M, Revil A (2010) Electrochemical charge of silica surfaces at high ionic strength in narrow channels. J Colloid and Int Sci 343:381-386

Xuan XC, Xu B et al (2005) Accelerated particle electrophoretic motion and separation in converging-diverging microchannels. Anal Chem 77(14):4323-4328

Xuan XC, Raghibizadeh S et al (2006) Wall effects on electrophoretic motion of spherical polystyrene particles in a rectangular poly (dimethylsiloxane) microchannel. J Colloid Interface Sci 296(2):743-748

Yariv E, Brenner H (2002) The electrophoretic mobility of an eccentrically positioned spherical particle in a cylindrical pore. Phys Fluids 14(9):3354-3357

Yariv E, Brenner H (2003a) The electrophoretic mobility of a closely fitting sphere in a cylindrical pore. Siam J Appl Math 64(2):423441

Yariv E, Brenner H (2003b) Near-contact electrophoretic motion of a sphere parallel to a planar wall. J Fluid Mech 484:85-111

Ye C, Li D (2004) Electrophoretic motion of two spherical particles in a rectangular microchannel. Microfluid Nanofluid 1(1):52-61

Ye CZ, Sinton D et al (2002) Electrophoretic motion of a circular cylindrical particle in a circular cylindrical microchannel. Langmuir 18(23):9095-9101

Ye CZ, Xuan XC et al (2005) Eccentric electrophoretic motion of a sphere in circular cylindrical microchannels. Microfluid Nanofluid 1(3):234-241 